\title{
Increased analgesia administration in emergency medicine after implementation of revised guidelines
}

\author{
Geesje Van Woerden', Crispijn L. Van Den Brand', Cornelis F. Den Hartog ${ }^{2}$, Floris J. Idenburg ${ }^{3}$, \\ Diana C. Grootendorst ${ }^{4}$ and M. Christien Van Der Linden ${ }^{1 *}$
}

\begin{abstract}
Background: The most common complaint of patients attending the emergency department (ED) is pain, caused by different diseases. Yet the treatment of pain at the ED is suboptimal, and oligoanalgesia remains common. The objective of this study is to determine whether the administration of analgesia at the ED increases by implementation of revised guidelines in pain management.

Methods: We conducted a prospective pre-post intervention cohort study with implementation of a revised guideline for pain management at our ED, in which nurses are allowed to administer analgesia (including lowdosage piritramid (opioid) intravenous) without doctor intervention. Numeric Rating Scales (NRS) were measured, and administration of medication (main outcome) was documented. We included every adult patient presenting with pain (NRS 4-10) at the ED.

Results: A total of 2107 patients (1089 pre-implementation phase and 1018 post-implementation phase) were included in our study. During pre-implementation, $25.4 \%$ of the patients with NRS between 4 and 10 received analgesia. After implementation, $32.0 \%$ of these patients received analgesia $(p<0.001)$.

Conclusions: After implementation of the revised guidelines in pain management at the ED, the administration of pain medication increased significantly. Nevertheless, the percentage of patients in pain receiving analgesia remain low (32\% after implementation).
\end{abstract}

Keywords: Analgesics, Pain management, Acute pain, Emergency department

\section{Background}

The most common complaint of patients attending the emergency department (ED) is pain, caused by different diseases. The pain prevalence in EDs, throughout the world, ranges from 52 to $79 \%$ [1]. Yet, the treatment of pain at the ED is suboptimal and oligoanalgesia remains common [2]. There are several factors contributing to this oligoanalgesia. First, the Numeric Rating Scale (NRS) to categorise pain as rated by physicians and nurses are both significantly lower than those reported by the patients $[3,4]$. Lack of knowledge and lack of guidelines in pain management also contribute to the

\footnotetext{
* Correspondence: c.van.der.linden@mchaaglanden.nl

${ }^{1}$ Emergency Department, Medical Centre Haaglanden, P.O. Box 4322501CK,

The Hague, The Netherlands

Full list of author information is available at the end of the article
}

inadequate treatment of pain [5]. Then, the manner in which a patient expresses pain is partly influenced by personality and culture, which is not always appreciated by a nurse or physician [6]. Lastly, the workload on the ED is high: nurses and physicians are caring for acutely ill patients in addition to patients in pain results in a lack of time to treat the pain properly [7].

\section{Importance}

Thus, improvement of knowledge (among nurses and doctors) and revision of guidelines may increase the administration of analgesia.

Therefore, the objective of the present study is to determine whether the administration of analgesia at the ED increases by implementation of revised guidelines in

\section{Springer}


pain management. Secondly, we will investigate which factors are contributing to pain management at the ED.

\section{Methods}

\section{Study design}

This was a prospective pre-post intervention cohort study with implementation of a revised guideline for pain management to increase the administration of analgesia. The study was divided into two periods (both 1 month) of data collection, before and after implementation of the revised guideline separated by a 1-month interval. During this interval, the revised guideline, developed by the emergency department (emergency physician and nurse) in cooperation with the anaesthesiology and consented by the specialties surgery and internal medicine, was implemented.

\section{Setting}

The study took place in the ED of a level 1 teaching (inner city) hospital and trauma centre with \pm 50.000 ED visits per year (Medical Centre Haaglanden). The study was conducted between September 2012 and January 2013. Our study was approved by the review board of the Medical Centre Haaglanden and by the Dutch Association of Medical Research Ethics Committee.

\section{Selection of participants}

We included every adult patient (16 years and older) presenting with pain (NRS 4-10) at the ED. As patients entered the ED, nurses assessed patients' NRS and entered the values into the patients' charts. Patients with life-threatening diseases/injuries requiring immediate transfer to the operating room or intensive care unit, altered mental status (Glasgow Coma Scale <14) and language barrier were excluded.

\section{Intervention: revision and implementation of guideline for pain management}

A clinical committee was formed, in which hospital staff from the emergency, anaesthesiology and surgical departments were represented. They reviewed the literature and revised the existing guideline for pain management according to the current standard [8]. In comparison to the previous guideline, nurses were allowed to administer analgesia (including low-dosage piritramid (opioid) intravenous (i.v.) without doctor intervention). The revised guideline included the following medication: acetaminophen, NSAID, tramadol, piritramid. Patients with pain NRS $>4$ were offered pain medication, with higher NRS indicating stronger medication (e.g. patient with NRS $=4-6$ were treated initially with acetaminophen per os (p.o.) whereas patient with NRS 7-10 were treated with piritramid i.v.).
The guideline was written in Dutch and distributed to all ED staff and nurses. The ED staff was informed by email. In addition, the ED nurses were also educated face-to-face. Pocket-sized versions (Fig. 1) of the guidelines were distributed, and posters were placed in the ED.

\section{Methods and measurements}

Demographics, pain assessment (NRS) at entrance and leave of the ED, administration of analgesia during ED visit, triage category, medical specialty and usage of analgesia before entering the ED (by patients at home or by EMS personnel) were retrieved from the medical records. Triage levels were assigned according to the fivelevel Manchester Triage System (MTS) [9]: 1 immediate, 2 very urgent, 3 urgent, 4 standard, and 5 non-urgent. In the analysis, triage levels were clustered because of small numbers of patients with levels 1 and 5, to high (categories 1 and 2), middle (category 3 ) and low (categories 4 and 5). Triage nurses used the pain ruler of the MTS in obtaining the level of urgency. In addition to the use of the MTS pain ruler, each patient is assessed with the NRS to measure the patients' actual pain level at entrance and leave of the ED. These NRS scores were used for this study. Medical specialty was divided into two categories: surgical specialty and non-surgical specialty. Surgical specialisms were dermatology, otorhinolaryngology, gynaecology, neurosurgery, ophthalmology, orthopaedics, plastic surgery, surgery, and urology. Nonsurgical specialties were cardiology, gastroenterology, internal medicine, neurology, pulmonology, and rheumatology. Patients were managed by emergency physicians as well as by residents. Data collection was conducted from the medical records of the included participants.

\section{Outcome measures}

The main outcome was administration of analgesia in general during ED visit. Secondly, we investigated patient and visit factors that influenced analgesic administration.

\section{Analysis}

Categorical data were expressed as number (\%) and compared using the $\chi^{2}$ test. Continuous data were analysed with a $t$ test. A $p$ value $<0.05$ was considered to be significant. The difference in analgesia administration before and after implementation was analysed by multivariate logistic regression analysis, adjusting for the following variables: triage category, usage of analgesia pre-hospital, ethnicity (Dutch versus other ethnicity), gender and age. According to the literature, all these factors are possible confounders in analysing the administration of pain medication [10-18]. We added the variables that were univariately associated with analgesic 


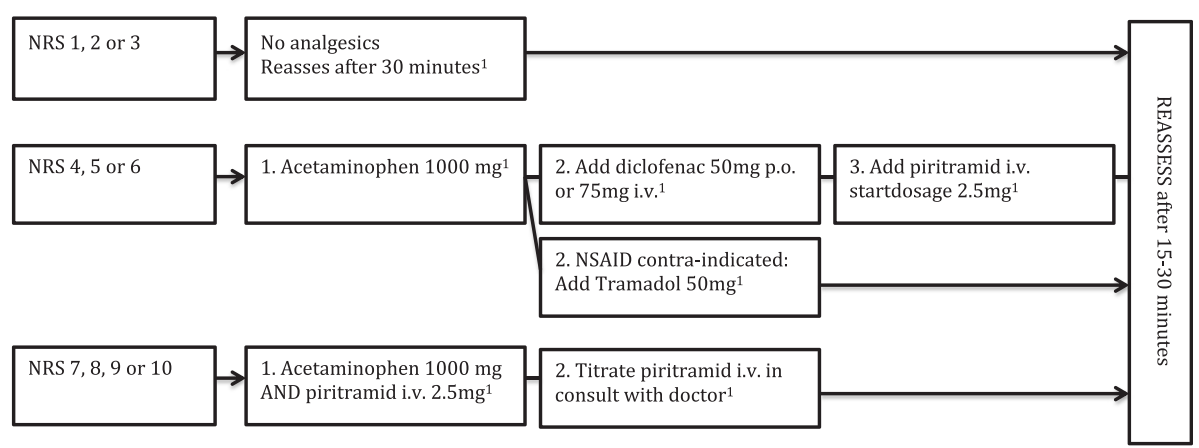

Fig. 1 Flowchart pain management. 'Reassess after each step, and if NRS is still $\geq 4$, follow next step in the flowchart

administration at $<0.05$. We created our model by the combination of the factors found in literature and the significant factors in our study and adjusted the analysis for these potential confounders. Effect sizes were expressed in adjusted odds ratios (ORs). Statistical uncertainty was expressed in a $95 \%$ confidence interval (CI). Statistical Package for the Social Sciences (SPSS) version 20 was used for all analyses.

\section{Results}

A total of 2107 patients (1089 resp. 1018) were included in our study (Fig. 2).

\section{Characteristics of study subjects}

Patients enrolled in the two phases of the study were similar with regard to age, gender and ethnicity (Dutch or other). After implementation, there were less patients in the high-NRS group. However, median NRS at admission was comparable between the two time periods. There were differences with regard to the use of analgesia pre-hospital and medical specialty: after implementation, significantly more patients were referred to surgical specialty, and furthermore, the use of analgesia pre-hospital was significantly lower (Table 1).

\section{Main results}

During the first phase of the study, $25.4 \%$ of the patients with a pain NRS between 4 and 10 received analgesia. After implementation, $31.7 \%$ of the patients in NRS 4-10 received analgesia $(p=0.001)$. The odds to receive analgesia after implementation was 1.35 higher than that before implementation (Table 2). After implementation, patients with a pain NRS between 7 and 10 more often received analgesics than patients with a pain NRS of $4-6$ (44 versus $25.7 \%, \chi^{2}<0.001$ ). After implementation the administration of analgesia was significantly increased in both NRS groups (NRS 4-6: OR 1.41 (1.09-1.83); NRS 7-10: OR 1.43 (1.04-1.96)). There was a significant difference in type of drugs administered after implementation: more acetaminophen was used and combinations of drugs were used more frequently during the post-implementation phase. The route of

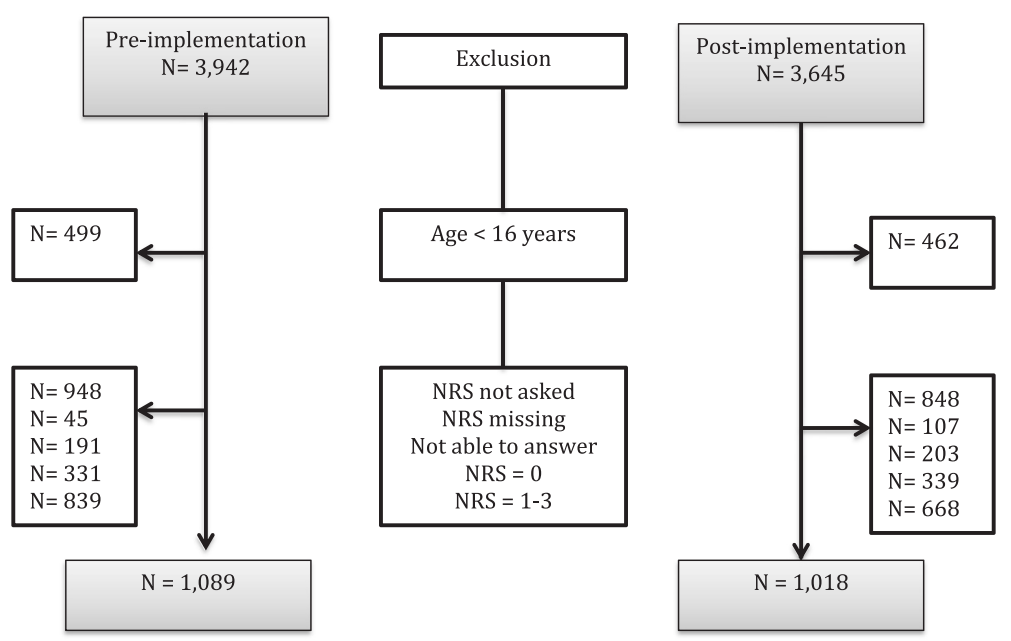

Fig. 2 Flow diagram in pre- and post-implementation phases 
Table 1 Patient characteristics pre- and post-implementation

\begin{tabular}{|c|c|c|c|}
\hline & Pre-implementation $(n=1089)$ & Post-implementation $(n=1018)$ & $p$ value \\
\hline & Mean years (lowest-highest) & Mean years (lowest-highest) & \\
\hline \multirow[t]{2}{*}{ Age } & $42(16-101)$ & $43(18-94)$ & 0.86 \\
\hline & Median (std. dev.) & Median (std. dev.) & \\
\hline \multirow[t]{2}{*}{ NRS at entrance ED (std. dev.) } & $5.0(1.7)$ & $5.0(1.6)$ & 0.086 \\
\hline & $N(\%)$ & $N(\%)$ & \\
\hline Male & $526(48.3)$ & $482(47.3)$ & 0.66 \\
\hline Dutch & $472(43)$ & $403(40.0)$ & 0.39 \\
\hline Other & $452(42)$ & $419(41.2)$ & \\
\hline Surgical specialty & $386(35.4)$ & $405(39.8)$ & 0.045 \\
\hline Non-surgical specialty & $700(64.3)$ & $613(60.2)$ & \\
\hline Triage category high & $171(15.7)$ & $153(15.0)$ & 0.003 \\
\hline Triage category middle & $509(46.7)$ & $547(53.7)$ & \\
\hline Triage category low & 409 (37.6) & $318(31.2)$ & \\
\hline Use of analgesia pre-hospital & $150(13.8)$ & $113(11.0)$ & 0.001 \\
\hline NRS 4-6 & $698(64.1)$ & $697(68.5)$ & $<0.001$ \\
\hline NRS 7-10 & $391(35.9)$ & $321(31.5)$ & \\
\hline
\end{tabular}

P-values printed in italic are statistically significant

administration also changed significantly: after implementation, more patients received medication p.o. (comparable with the difference in the administered acetaminophen). There was no difference in the medication given intravenously (Table 3).

In both pre- and post-implementation, several factors contributed to the administration of analgesia. Firstly, the higher triage categories received more frequent analgesia $(\mathrm{OR}$ for the highest triage category $=3.98,95 \% \mathrm{CI}$ $2.86-5.54$, in which the lowest triage category is the reference category). Secondly, patients treated by a surgical specialty received more frequent analgesia than patients treated by a non-surgical specialty (OR 2.47, $95 \% \mathrm{CI}$ 1.98-3.09) (Table 3). This second finding was independent of NRS at entrance ED (OR 2.32, 95 \% CI 1.85-2.91; adjusted for variables in Table 2). Age, gender and

Table 2 Patient and visit factors that influence analgesic administration

\begin{tabular}{lllll}
\hline & Crude OR & $95 \% \mathrm{Cl}$ & Adjusted $\mathrm{OR}^{*}$ & $95 \% \mathrm{Cl}$ \\
\hline $\begin{array}{l}\text { Implementation of } \\
\text { revised guideline for } \\
\text { pain management }\end{array}$ & 1.35 & $1.13-1.65$ & 1.35 & $1.11-1.65$ \\
$\begin{array}{l}\text { Triage category } \\
\quad\end{array}$ & & & & \\
$\quad$ Low = reference & & & & \\
$\quad$ Middle & 2.40 & $1.91-3.01$ & 3.00 & $2.36-3.82$ \\
$\quad$ High & 2.42 & $1.79-3.26$ & 3.98 & $2.86-5.54$ \\
Surgical specialty & 1.75 & $1.43-2.15$ & 2.47 & $1.98-3.09$ \\
\hline
\end{tabular}

*Included in the model were medical specialty (surgical versus non-surgical), triage category, usage of analgesia pre-hospital, ethnicity (Dutch versus other ethnicity), gender and age. Only significant variables are shown in the table ethnicity were not significantly correlated to the administration of analgesia.

In a certain number of patients in both groups (before and after implementation), the pain NRS at entrance to the ED was missing, either because it was not established or the patient was unable to answer (Fig. 2). Among these patients, 10 versus $12.7 \%$ (before and after) received analgesics, and more patients were categorised as low urgency by triage compared to the total research population. NRS at leave of the ED was missing in $46 \%$ of the patients ( $n=973)$.

\section{Discussion}

This study has several limitations. First, it was a large prospective observational study. Therefore, we can only show associations and no causality. We could not abstract from the charts whether the analgesic administration was protocol driven or by treating physicians. We did not use a blind design because we aimed to modify clinical practice of the ED staff by implementing the revised guidelines. During this period, there were no other changes in pain management (e.g. change in immobilisation, regional blocks), so the effect observed in this prepost study was likely to be caused by the intervention.

Second, there is a percentage of patients (in both groups) whose pain NRS at entrance to the ED was not measured. Compared to the research group, this group was triaged as less urgent and they received fewer analgesics. We assume that this group was treated during triage and left the ED before being asked the pain NRS. Since the group was triaged as low urgency, the NRS scores are expected to be low (e.g. in case of moderate 
Table 3 Administration of analgesics: drug types and administration routes

\begin{tabular}{llll}
\hline & $\begin{array}{l}\text { Pre- } \\
\text { implementation } \\
N(\%)\end{array}$ & $\begin{array}{l}\text { Post- } \\
\text { implementation } \\
N(\%)\end{array}$ & $\begin{array}{l}p \\
\text { value }\end{array}$ \\
\hline $\begin{array}{l}\text { Administration of } \\
\text { analgesics }\end{array}$ & $277(25.4)$ & $323(31.7)$ & 0.001 \\
$\begin{array}{l}\text { Drug types } \\
\text { Acetaminophen }\end{array}$ & $175(16.1)$ & $204(20.0)$ & \\
$\quad$ NSAID & $32(2.9)$ & $19(1.9)$ & 0.018 \\
$\begin{array}{l}\text { Tramadol } \\
\text { Piritramid }\end{array}$ & $4(0.4)$ & $4(0.4)$ & 0.11 \\
$\begin{array}{l}\text { Drug } \\
\text { combination }\end{array}$ & $51(0.5)$ & $2(0.2)$ & 0.92 \\
Administration & & $94(9.2)$ & 0.30 \\
routes & & & 0.001 \\
By mouth & $171(15.7)$ & $208(20.4)$ & 0.005 \\
Intravenous & $106(9.7)$ & $115(11.3)$ & 0.24 \\
\hline
\end{tabular}

${ }^{\mathrm{a}}$ Combination of drugs mentioned above (acetaminophen, NSAID, tramadol, piritramid)

P-values printed in italic are statistically significant

or severe pain, the triage category is automatically higher), and therefore, it is possible that a large amount of this group would have been excluded (NRS <4). Thus, we assume the effect of our intervention would not change if we had measured the NRS in this group. There are also high urgency patients in this group. An explanation for the missing NRS in this subgroup could be that their injuries/diseases were of such urgency that they had to be transferred to the operation room or intensive care unit immediately. If we had been able to measure the NRS in this high urgency (with expected high NRS scores) category, one could expect the effect of our intervention to be the same or even larger, because analgesic administration is significantly associated with increasing severity of pain $[19,20]$.

Third, the Hawthorne effect (change of behaviour of nurses and physicians induced by the study itself) is difficult to avoid. Yet, the effect would have been present in both study phases.

Fourth, the use of analgesia pre-hospital was higher before implementation than after implementation. To avoid overdoses, one could expect that less analgesia was administered in the pre-implementation group or more analgesia was administered in the post-implementation group. However, we adjusted the analysis for the use of pre-hospital analgesia and still the difference between the administration of analgesia between pre- and postimplementation was significant.

Fifth, we were not able to measure ethnicity properly, rather than dividing patients into Dutch and not-Dutch based on country of birth of the patient and his or her parents. This may have caused biased information.
Sixth, at baseline, there were differences between the two groups in triage category. Yet, this difference is not explained by more pain, because the median NRS is comparable in the two groups. Although the nurses are obligated to register the administration of medication, it was possible to administer the medication but forget to register this in the patient chart. Thus, the effect size is possibly underestimated.

Finally, the pre- and post-groups differed with respect to specialty and triage category. Therefore, the finding that patients with surgical problems received more often analgesia may have been biased. Still, patients admitted to the ED with a non-surgical problem received less often pain medication than the patients treated by one of the surgical specialties, independent of triage category. Since the education on pain management for all emergency department staff did not differ, this could not explain the found difference. One hypothesis that would explain the difference in specialty in the administration of analgesia would be that tangible proof (e.g. wound, radiographic findings) influences physician/nurse practice more than patients' reports of pain.

In this study, we demonstrated that after implementation of the revised guideline in pain management at the ED, the administration of pain medication increases significantly. Nevertheless, the percentage of patients in pain receiving analgesia remain low (32\% after implementation). In line with this main outcome, Decosterd et al. [5] showed that the number of patients receiving analgesia increased significantly after implementation of the guidelines. Yet, the increase achieved in their study was more extensive (63\% of the patients received analgesic postimplementation). The smaller effect in our study might be explained by the differences in baseline NRS scores. Though the median NRS was comparable, there were less patients in the post-group with higher NRS scores, possibly leading to less analgesics administration [20].

In our study, inadequate pain management is evident. Several other studies show that around $40 \%$ of patients in pain at the ED do not receive pain medication [21, 22]. This indicates that in this study, oligoanalgesia was and still is a problem. We only measured the administration of analgesics at the ED. The real important issue is whether the pain relief administered has been effective or not [23]. However, in our research, $46 \%$ of these NRS scores at leave were missing, so we were not able to measure effectiveness of administration of analgesics.

To address the differences in pain medication administration, this study found that age was not correlated to the administration of analgesics. Previous research is contradictory: according to Jones et al. [24], elderly 
patients receive less pain medication whereas a more recent study of Cinar [25] did not find any correlation between age and administration of analgesics. Similar results are known for ethnicity. In our study, we did not find a correlation between ethnicity and administration of analgesics, a finding supported by some literature but refuted by other sources which state that non-white patients are less likely to be treated for pain $[10,12,14,17,18]$.

According to our study, male and female patients had similar chance of being treated with pain medication. Raftery et al. [26], on the other hand, showed that female patients reported higher pain scores and received more pain medication.

In the future, we would like to test the hypothesis that tangible proof causes differences in the management of pain. There is a high variability in pain management frameworks and protocols among EDs worldwide [27]. The use of our nurse-driven protocol including opioid analgesics based on a pain rating scale has not been validated yet. Since oligoanalgesia is still a major problem in our ED, identifying barriers to ED triage nurses' management of patients' pain is important. Also, although less of an issue in The Netherlands, there are concerns of misuse and abuse of opioid analgesics in some other parts of the world $[28,29]$. A future study regarding the usage of opioids in Dutch ED triage practice would be of interest. Going forward, we would like to implement a NRS reassessment after the administration of analgesia to measure the effect of the medication. Lastly, a longer-term study into adherence to the new guideline is of interest.

\section{Conclusions}

In summary, we found that after implementation of the revised guidelines in pain management at the ED, the administration of pain medication increased significantly. Nevertheless, the percentage of patients in pain which receive analgesia remain low (32\% after implementation). We also found that triage category and surgical specialty were influencing the administration of pain medication.

\section{Abbreviations}

Cl: confidence interval; ED: emergency department; i.v.: intravenous; MTS: Manchester Triage System; NRS: Numeric Rating Scale; OR: odds ratio; p.o.: per os; SPSS: Statistical Package for the Social Sciences.

\section{Competing interests}

The authors declare that they have no competing interests.

\section{Authors' contributions}

GW, ML, CB conceived the study and designed the trial. GW and ML supervised the conduct of the trial and data collection. GW and $\mathrm{CH}$ revised the former guideline in pain management. ML and DG provided statistical advice on the study design. GW analysed the data. GW drafted the manuscript. All authors contributed substantially to its revision. GW takes responsibility for the paper as a whole. All authors read and approved the final manuscript.

\section{Author details}

${ }^{1}$ Emergency Department, Medical Centre Haaglanden, P.O. Box 4322501CK, The Hague, The Netherlands. ${ }^{2}$ Department of Anaesthesiology, Medical Centre Haaglanden, P.O. Box 4322501CK, The Hague, The Netherlands. ${ }^{3}$ Department of Surgery, Medical Centre Haaglanden, P.O. Box $4322501 \mathrm{CK}$, The Hague, The Netherlands. ${ }^{4}$ Landsteiner Institute, Medical Centre Haaglanden, P.O. Box 432, 2501CK, The Hague, The Netherlands.

Received: 19 December 2014 Accepted: 3 February 2016

Published online: 10 February 2016

\section{References}

1. Berben SA, Meijs TH, van Dongen RT, van Vugt AB, Vloet LC, Mintjes-de Groot JJ, et al. Pain prevalence and pain relief in trauma patients in the Accident \& Emergency department. Injury. 2008;39:578-85.

2. Todd KH, Ducharme J, Choiniere M, Crandall CS, Fosnocht DE, Homel P, et al. Pain in the emergency department: results of the pain and emergency medicine initiative (PEMI) multicenter study. J Pain. 2007;8:460-6.

3. Carter D, Sendziuk P, Eliott JA, Braunack-Mayer A. Why is pain still undertreated in the emergency department? Two new hypotheses. Bioethics. 2015. PMID:26104124 [Epub ahead of print]. doi:10.1111/bioe.12170.

4. Guru V, Dubinsky I. The patient vs. caregiver perception of acute pain in the emergency department. J Emerg Med. 2000;18:7-12.

5. Decosterd I, Hugli O, Tamches E, Blanc C, Mouhsine E, Givel JC, et al. Oligoanalgesia in the emergency department: short-term beneficial effects of an education program on acute pain. Ann Emerg Med. 2007;50:462-71.

6. Rupp T, Delaney KA. Inadequate analgesia in emergency medicine. Ann Emerg Med. 2004;43:494-503.

7. Pretorius A, Searle J, Marshall B. Barriers and enablers to emergency department nurses' management of patients' pain. Pain Manag Nurs. 2015; 16:372-9.

8. Dutch Association of Emergency Nurses. Dutch guideline: pain management in emergency medicine for trauma patients. 2010.

9. Mackway-Jones K, Marsden J, Windle J. Emergency triage. London: BMJ Publishing Group; 2005.

10. Choi DM, Yate P, Coats T, Kalinda P, Paul EA. Ethnicity and prescription of analgesia in an accident and emergency department: cross sectional study. BMJ. 2000;320:980-1.

11. Daoust R, Paquet J, Piette E, Sanogo K, Bailey B, Chauny JM. Impact of age on pain perception for typical painful diagnoses in the emergency department. J Emerg Med. 2016;50(1):14-20.

12. Johnson TJ, Weaver MD, Borrero S, Davis EM, Myaskovsky L, Zuckerbraun NS, et al. Association of race and ethnicity with management of abdominal pain in the emergency department. Pediatrics. 2013;132:e851-8.

13. Marco CA, Nagel J, Klink E, Baehren D. Factors associated with self-reported pain scores among ED patients. Am J Emerg Med. 2012;30:331-7.

14. Motov SM, Khan AN. Problems and barriers of pain management in the emergency department: are we ever going to get better? J Pain Res. 2008:2:5-11.

15. Musey Jr PI, Linnstaedt SD, Platts-Mills TF, Miner JR, Bortsov AV, Safdar B, et al. Gender differences in acute and chronic pain in the emergency department: results of the 2014 Academic Emergency Medicine consensus conference pain section. Acad Emerg Med. 2014;21:1421-30.

16. Platts-Mills TF, Esserman DA, Brown DL, Bortsov AV, Sloane PD, McLean SA. Older US emergency department patients are less likely to receive pain medication than younger patients: results from a national survey. Ann Emerg Med. 2012;60:199-206.

17. Tamayo-Sarver JH, Hinze SW, Cydulka RK, Baker DW. Racial and ethnic disparities in emergency department analgesic prescription. Am J Public Health. 2003;93:2067-73.

18. Todd KH, Samaroo N, Hoffman JR. Ethnicity as a risk factor for inadequate emergency department analgesia. JAMA. 1993;269:1537-9.

19. Kuan SC, Collins NC, Ryan JM, Callanan I. Treating pain in the emergency department. Eur J Emerg Med. 2010;17:52-5.

20. Rahman NH, Ananthanosamy C. The display effects of patients' selfassessment on traumatic acute pain on the proportion and timing of analgesics administration in the emergency department. Int J Emerg Med. 2014;7:36 
21. Berben SA, Schoonhoven L, Meijs TH, van Vugt AB, van Grunsven PM. Prevalence and relief of pain in trauma patients in emergency medical services. Clin J Pain. 2011;27:587-92.

22. Wilson JE, Pendleton JM. Oligoanalgesia in the emergency department. Am J Emerg Med. 1989;7:620-3.

23. Stang AS, Hartling L, Fera C, Johnson D, Ali S. Quality indicators for the assessment and management of pain in the emergency department: a systematic review. Pain Res Manag. 2014;19:e179-90.

24. Jones JS, Johnson K, McNinch M. Age as a risk factor for inadequate emergency department analgesia. Am J Emerg Med. 1996;14:157-60.

25. Cinar O, Ernst R, Fosnocht D, Carey J, Rogers L, Carey A, et al. Geriatric patients may not experience increased risk of oligoanalgesia in the emergency department. Ann Emerg Med. 2012;60:207-11.

26. Raftery KA, Smith-Coggins R, Chen AH. Gender-associated differences in emergency department pain management. Ann Emerg Med. 1995;26:414-21.

27. Parnass AJ, Greenbaum NR, Glick MA, Halpern P. Pain management framework in the emergency department: patterns in 40 emergency departments worldwide. Eur J Emerg Med. 2015. PMID: 26225613 [Epub ahead of print].

28. Beaudoin FL, Straube S, Lopez J, Mello MJ, Baird J. Prescription opioid misuse among ED patients discharged with opioids. Am J Emerg Med. 2014;32:580-5.

29. West NA, Severtson SG, Green JL, Dart RC. Trends in abuse and misuse of prescription opioids among older adults. Drug Alcohol Depend. 2015;149:117-21.

\section{Submit your manuscript to a SpringerOpen ${ }^{\odot}$ journal and benefit from:}

- Convenient online submission

- Rigorous peer review

- Immediate publication on acceptance

- Open access: articles freely available online

- High visibility within the field

- Retaining the copyright to your article

Submit your next manuscript at $\gg$ springeropen.com 